\title{
On the Structure and Synaptic Organization of the First Optic Ganglion in the Fly
}

\section{BRUCE BosCHeK}

Max-Planck-Institut für biologische Kybernetik Tübingen

(Z. Naturforsch. 25 b, 560 [1970] ; eingegangen am 25. März 1970)

The lamina ganglionaris is the first synaptic region in the compound eye of the fly. Presumed synaptic sites have been observed within the optical cartridges in the external plexiform layer and are characterized by the presence of T-shaped synaptic ribbons within the presynaptic fibers ${ }^{1}$. Each optical cartridge was shown to be composed of two centrally located axons of second order monopolar neurons (type I) surrounded by a crown of six axon terminals belonging to retinula cells no. 1 to 6 . Each one of the retinula cell axons is associated with a pair of centrifugal fiber endings. Type II neurons were also observed but the fate of their processes remained uncertain. The six photoreceptor axons provided synaptic inputs to both central second order neurons. The centrifugal fiber endings were also described as presynaptic to the second order neurons as well as to the six photoreceptor axon endings. The axons of the two photoreceptors, no. 7 and no. 8, were not observed to make synaptic contact in the lamina.

The present investigation was performed using the electron microscope and the technique of serial sectioning in order to determine more completely the structure of the optical cartridges as well as to develop a synaptic diagram containing as many synapses as could be found in the lamina. The compound eyes of adult female specimens of Musca domestica were studied.

Fig. 1 summarizes the structure and synaptic interconnections of the optical cartridges. Two thin fibers which can be seen to originate from two type II somata are located on the periphery of the cartridge. One of these corresponds to $\mathrm{L}_{3}$, the other one to $\mathrm{L}_{4}$ in the terminology of BRAITENBERG and StRAusfeld ${ }^{2}$.

The most prevalent type of synapse, already described by Trujillo-Cenóz ${ }^{1}$, occurs between $\mathrm{R}_{1-6}$ and $\mathrm{L}_{1}$ and $\mathrm{L}_{2}$ (synapse type 1 , fig. 1 ). $\mathrm{R}_{1-6}$ are also presynaptic to at least one of the paired centrifugal fibers $\alpha, \beta$ (synapse type 2), a result which is in direct opposition to previous reports of observations in other dipterans ${ }^{1}$, where ribbons were found within the centrifugal fibers at junctions with $R_{1-6}$. No such contacts have been observed in Musca. $\mathrm{L}_{3}$ receives synaptic input from at least one of the photoreceptor axons (synapse type 3 ), both $\mathrm{L}_{3}$ and $\mathrm{L}_{4}$ receive inputs from the centrifugal fibers (type 4 ). $\mathrm{L}_{4}$ has a short side branch which re-enters the parent cartridge at its base. It is still uncertain whether this side branch is responsible for the fiber fragment seen to make the type 5 synapse. The centrifugal fibers make contact with one another as well as with small unidentified fibers (types 6 and 7). Ribbons have been observed within $\mathrm{L}_{1}$ and $\mathrm{L}_{2}$ however the postsynaptic fiber has not yet been identified (type 8).

1 O. Trujillo-Cenóz, J. Ultrastruct. Res. 13, 1 [1965].

2 V. Brattenberg and N. Strausfeld, Handbook of Sensory Physiology, in print.

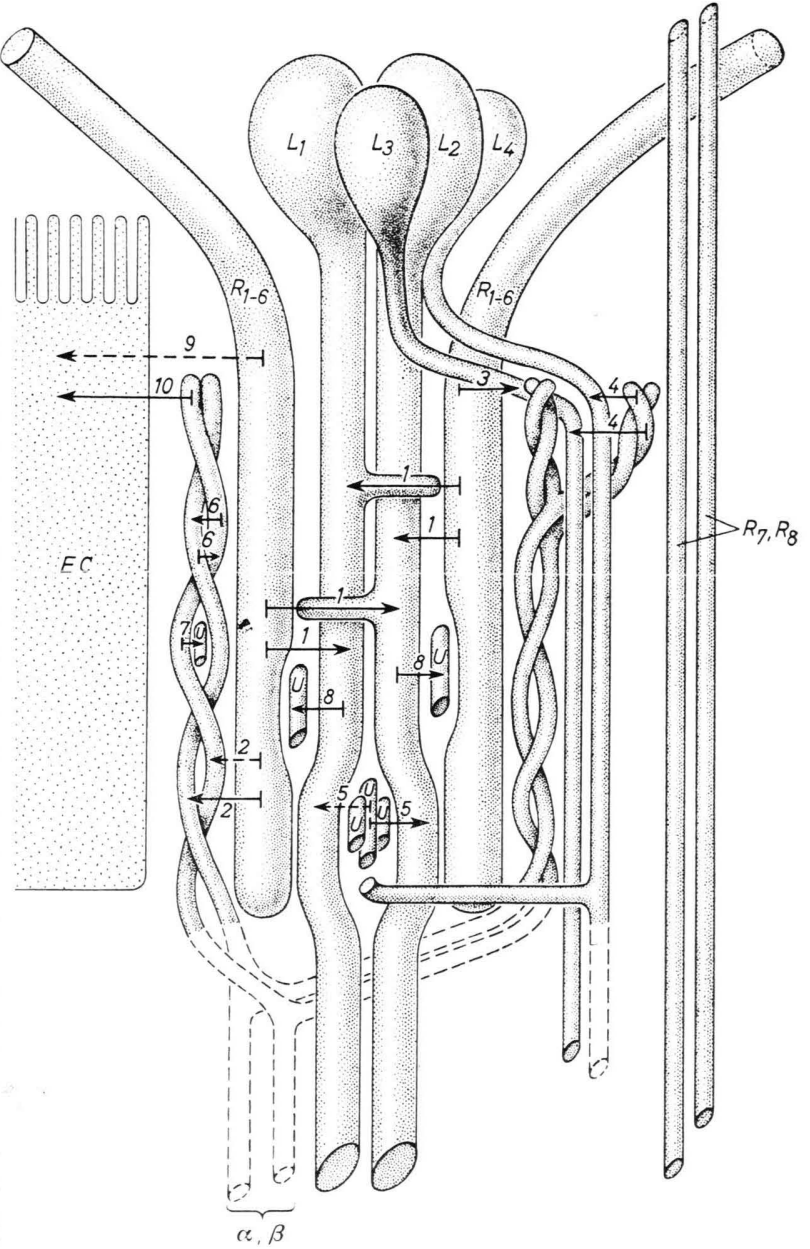

Fig. 1. Summary diagram of the structure and synaptic interconnections of the optical cartridges. For the sake of clarity only two of the six photoreceptor axon terminals $\left(R_{1-6}\right)$ have been shown. $\mathrm{R}_{7}, 8$, axons of the central photoreceptors en route. $\alpha, \beta$, paired centrifugal endings (again, only two of the six pairs have been drawn). $\mathrm{L}_{1}-\mathrm{L}_{4}$, monopolar neurons. EC, epithelial glial cell. U, unidentified fiber fragments. The arrows indicate synaptic polarity based on the assumption that the T-shaped synaptic ribbons occur only within the presynaptic fibers. The numbers are used for identification purposes in the text. Broken lines indicate areas of uncertainty. Semischematic, not to scale.

The arrows labelled 9 and 10 indicate that T-shaped ribbons are also observed with the epithelial glial cells as postsynaptic elements. These findings raise the question as to whether these junctions are synaptic in function and indicate the need for further investigation of the epithelial glial cell.

Fig. 1 shows that at least 8 fibers leave the cartridge. Five of them have been mentioned by HorRIDGE and Meinertzhagen in a recently published paper ${ }^{3}$.

I am most grateful to Dr. Braitenberg, Dr. Kirschfeld and Dr. STRAusfeld for valuable discussions.

${ }^{3}$ G. A. Horridge and I. A. Meinertzhagen, Z. vergl. Physiol. 66, 369 [1970]. 